\title{
A Haskell Hosted DSL for Writing Transformation Systems
}

\author{
Andy Gill \\ Information Technology and Telecommunication Center, \\ Department of Electrical Engineering and Computer Science, \\ The University of Kansas, \\ 2335 Irving Hill Road, \\ Lawrence, KS 66045 \\ andygill@ku.edu
}

\begin{abstract}
KURE is a Haskell hosted Domain Specific Language (DSL) for writing transformation systems based on rewrite strategies. When writing transformation systems, a significant amount of engineering effort goes into setting up plumbing to make sure that specific rewrite rules can fire. Systems like Stratego and Strafunski provide most of this plumbing as infrastructure, allowing the DSL user to focus on the rewrite rules. KURE is a strongly typed strategy control language in the tradition of Stratego and Strafunski. It is intended for writing reasonably efficient rewrite systems, makes use of type families to provide a delimited generic mechanism for tree rewriting, and provides support for efficient identity rewrite detection.
\end{abstract}

\section{Introduction}

Sometimes its easy to say what you want to do, but tedious to get the scaffolding in place. Such a situation is an ideal candidate for a Domain Specific Language (DSL) where the language provides the scaffolding as a pre-packaged service. For example, scaffolding is needed to promoting local, syntax directed rewrites into a global context. Take the first case rewriting rule from the Haskell 98 Report [1] which says:

(a) case e of $\{$ alts $\}=(\backslash \mathrm{v} \rightarrow$ case $\mathrm{v}$ of $\{$ alts $\}) e$ where $v$ is a new variable

This rule is used to simplify a case over an arbitrary expression, and convert it to a case over a variable. We can express this rule directly in Haskell, using a rewrite in the Haskell Syntax provided by Template Haskell [2, using the function rule_a.

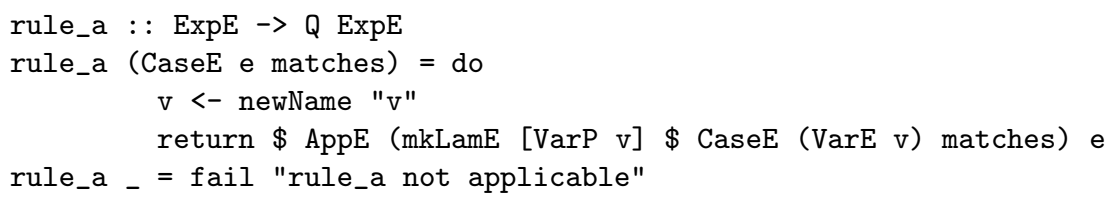


This rule reflects our syntax rewrite rule almost directly, and cleanly utilizing a monadic name supply for a fresh variable. The rule also acts locally, and needs additional machinery to perform this rewrite on whole programs. Strategic programming is a paradigm which builds on rewrite primitives like rule_a by using combinators to construct complex and powerful rewrites and transformation systems.

This paper introduces KURE, a strategic programming DSL hosted in Haskell being developed at the University of Kansas. KURE provides a small set of combinators that can be used to build parameterized term rewriting and general user-defined rule application. In this paper, we discuss in detail the design of KURE, and how we used the tradition of type-centric DSL design to drive our implementation. This paper makes the following specific contributions.

- We move both rewrites and rewrite strategies into a strongly typed world. Throughout the paper we make detailed comparisons with previous attempts to providing typing to rewrite strategy systems.

- More specifically, we provide typing for term traversing strategies using a new capability, a lightweight and customized generic term traversal mechanism implemented using associated type synonyms [3].

- We introduce the ability to record equality over terms, which is traditionally a weakness of purely functional rewrite engines. Equality is important because the use-case for many transformations in practice is to iterate until nothing else can be achieved.

- We abstract our combinators explicitly over a user-defined context, allowing concerns like environment or location to be represented. We show the generality of this contribution by implementing path selection. This design choice allows a particularly clean relationship between the semantics being hosted by the DSL, and the implementation using our DSL.

- We lay out the methodology used in the design of our hosted DSL, with the intent that others can use our design template.

KURE builds on many years of research and development into rewrite strategies, especially the work of Stratego [4] and Strafunski [5], and our own experiences with HERA [6]. We compare KURE to these systems throughout this paper, as well as make different design decisions explicit, and summarize the differences in section 11. In recognition of the contributions made by these systems, we reuse where possible the naming conventions of Stratego and Strafunski.

\section{Introducing Strategic Programming}

Stratego and other strategic programming languages provide a mechanism to express rewrites as primitive strategies, and a small set of combinators that act on strategies, giving the ability to build complex custom strategies. In this section, we introduce the basic combinators of strategic programing used in Stratego. This will guide our design of KURE. 
Table 1. Combinators in Stratego

\begin{tabular}{ll}
\hline Combinator & Purpose \\
\hline id & identity strategy \\
fail & always failing strategy \\
$\mathcal{S}<+\mathcal{S}$ & local backtracking \\
$\mathcal{S} ; \mathcal{S}$ & sequencing \\
all $(\mathcal{S})$ & apply $\mathcal{S}$ to each immediate child \\
\hline
\end{tabular}

A basic strategy is a rewrite from one abstract syntax term to another abstract syntax term, for example:

$$
\text { NOT1 : } \operatorname{Not}(\operatorname{Not}(e)) \rightarrow e
$$

This strategy, called NOT1, attempts to match a term, and if the top node is Not, and the immediate child is also Not, then Stratego replaces this whole term with the immediate child of the second Not and terminates successfully. If the term is not matched, the strategy fails. In functional parlance, strategies take a term, and return a new term, or fail.

As for parsing combinators [7, a rich algebra of combinators can be built on top of the concept of strategies. If $\mathcal{S}$ is a strategy, the key combinators in Stratego are listed in Table 1, id is the identity transformation, fail is a transformation that always fails, <+ is a choice operator with local backtracking, ';' sequences two transformations, and all provides a shallow traversal, a traversal of only the immediate children of a node. There are others, but these capture the spirit of the programming paradigm.

We can use these primitive combinators to write other combinators, like try

$$
\operatorname{try}(s)=s<+ \text { id }
$$

which attempts a rewrite, and if it fails, performs the identity rewrite instead. try has the nice property, therefore, that it never fails.

All of the combinators introduced so far are shallow, and only act on at most a single level of a term. We can use these to implement deeper rewrites, which act over every sub-node in a tree.

$$
\text { topdown }(s)=s ; \operatorname{all}(\operatorname{topdown}(s))
$$

Stratego also provides the ability to invoke a strategy from within a rule, by enclosing the strategy in angle brackets.

$$
\text { EvalAdd : Add }(\operatorname{Int}(i), \operatorname{Int}(j)) \rightarrow \operatorname{Int}(\langle\operatorname{addS}\rangle(i, j))
$$

Here, addS is itself a rewrite strategy which takes a 2-tuple of integers, and generates the result of the addition. 
By using application inside rules strategy-based programming jumps between rules and strategies, and back again. Basic user strategies are named rules, and rules can use strategies to express rewrites over terms, collectively forming a productive environment to implement complex rewrites. Critically, locally applicable rules are given the opportunity to act over many sub-terms in a controlled matter. Using this programming idiom several rewrite systems have been implemented on top of Stratego and related tools, including Stratego itself, optimizers, pretty printers, and COBOL engineering tools. The idiom has demonstrated the ability to have all the qualities of a great DSL, giving leverage to the rewrite author. We have only given a cursory overview of the essence of Stratego and strategic programming, and the interested reader is referred to the Stratego website, http://strategoxt.org/

When considering a rewriting system using strategies hosted in Haskell, Strafunski is currently the most mature library. Strafunski is a strategic programming implementation which follows in the tradition of Stratego, adds typing to primitive transformations, and uses "scrap your boilerplate" (SYB) generics [8] to implement shallow (single-level) and deep (multi-level) traversals over arbitrary term types. Strafunski provides both type-preserving rewrites and unifying rewrites (rewrites that map to a single common type). However, there are shortcomings that merit revisiting the design decisions of Strafunski. KURE is an attempt to revisit these design decisions. Specifically, KURE replaces the powerful hammer of SYB generics provided in Strafunski with a more precise, user configurable and lightweight generics mechanism. KURE also provides a number of parameterization opportunities over Strafunski, as well as other differences, as discussed in section 9 .

\section{Design of the KURE Kernel}

Our design and implementation of KURE follows the classical DSL hosted in Haskell approach, namely

- we propose specific functionality for the primitive combinators of our DSL,

- we unify the combinators around a small number of (typically abstract) types,

- we postulate the monad that is contained inside the computation of these primitives,

- we invent some structure around this monad, to provide the significant userlevel types in our DSL,

- and at this point, our combinators are largely implemented using routine plumbing between monadic computations.

There are structures other than monads that can be used to model computations, but monads are certainly the tool of choice in the Haskell community for modeling such things.

The major primitive combinators in a DSL for strategic programming are well understood, and have been discussed in section 2 . We want to add two 
new facilities, both of which have analogues in Stratego, but are implemented in a functional and strongly typed way in KURE. First, we add the ability to understand the context of a rule. Rather than introduce dynamically scoped rules 9] we want our rules to execute in a readable environment that reflects the context within which the rule is being executed. We also add the ability to create new global bindings from within local rules.

Most importantly, we want our DSL to use types to reflect the transformations taking place. The next section reviews how other systems have added types to strategic programming.

\section{Previous Uses of Types in Strategic Programming}

There have been a number of attempts to add typing to strategy based programming. There are two independent issues to resolve. Firstly, giving a type to a strategy $\mathcal{S}$. Secondly, giving general types to functions that do both shallow and deep rewrites, like all and topdown. We address each of these in turn.

\subsection{Giving a Type to $\mathcal{S}$}

Giving a type to $\mathcal{S}$ is straightforward. Consider a typed strategy or transformer, called $\mathcal{T}$. We can give $\mathcal{T}$ two type parameters, the initial term's type, and the type of the term after rewriting. Thus, our strategies have the type:

$$
\mathcal{T} t_{1} t_{2}
$$

We can now give types to the depthless combinators in Stratego, which we do in Table 2 .

Table 2. Types for Depthless Combinators

\begin{tabular}{lll}
\hline Combinator & \multicolumn{1}{c}{ Type } & \\
\hline id & $\forall t_{1}$. & $\mathcal{T} t_{1} t_{1}$ \\
fail & $\forall t_{1}, t_{2}$. & $\mathcal{T} t_{1} t_{2}$ \\
$\mathcal{S}<+\mathcal{S}$ & $\forall t_{1}, t_{2}$. & $\mathcal{T} t_{1} t_{2} \rightarrow \mathcal{T} t_{1} t_{2} \rightarrow \mathcal{T} t_{1} t_{2}$ \\
$\mathcal{S} ; \mathcal{S}$ & $\forall t_{1}, t_{2}, t_{3} . \mathcal{T} t_{1} t_{2} \rightarrow \mathcal{T} t_{2} t_{3} \rightarrow \mathcal{T} t_{1} t_{3}$ \\
\hline
\end{tabular}

For combinations of these combinators, the type system works unremarkably. For example, the function try can be given a straightforward type.

$$
\begin{aligned}
& \operatorname{try}:: \forall t_{1} \cdot \mathcal{T} t_{1} t_{1} \rightarrow \mathcal{T} t_{1} t_{1} \\
& \operatorname{try}(s)=s<+ \text { id }
\end{aligned}
$$

Transforms that are type-preserving are common in strategy based programming, so we give such transforms there own type name, $\mathcal{R}$.

$$
\mathcal{R} t_{1}=\mathcal{T} t_{1} t_{1}
$$


$\mathcal{R}$ is simply an abbreviation for $\mathcal{T}$ for notational convenience. Using $\mathcal{R}$, try can be giving the equivalent type

$$
\operatorname{try}:: \forall t_{1} \cdot \mathcal{R} t_{1} \rightarrow \mathcal{R} t_{1}
$$

Giving types to application and abstraction using $\mathcal{T}$ is straightforward once a monad for rules has been selected, so we defer giving the actual types until section 6 in the context of our KURE monad, and observe that there are no technical issues with their typing.

\subsection{Types for Shallow and Deep Combinators}

Adding types is more problematic for shallow and deep combinators. Consider the combinator all, which acts on immediate children, but leave the root node unmodified. This implies a type-preserving rewrite, and we would expect something of the form

$$
\text { all }:: \forall t_{1} . \mathcal{R} t_{1} \rightarrow \mathcal{R} t_{1}
$$

This combinator can only apply its argument to immediate children of the same type, a significant shortcoming, but is the approach taken by Dolstra [10, when he attempts to host strategies directly in Haskell.

If we give all a more general type, other issues surface

$$
\text { all :: } \forall t_{1}, t_{2} \cdot \mathcal{R} t_{1} \rightarrow \mathcal{R} t_{2}
$$

Unfortunately, the type $t_{1}$ is completely unrelated to $t_{2}$, so it is well understood that all can never actually use its argument in any interesting or non-trivial way, without runtime type comparisons. This is the the approach taken by Dolstra [1]; Dolstra and Visser 1112, who invent a new language with its own type system which includes runtime type-case internally inside all.

This is also essentially the approach taken in Strafunski [5]. Rather than work on polymorphic rewrites directly, Strafunski implements all by wrapping $\mathcal{R}$ in an abstraction TP. Here, we use the notation from [13] for expressing type contexts.

$$
\mathrm{TP}=\forall t_{1}\left\langle\text { Term } t_{1}\right\rangle \Rightarrow \mathcal{R} t_{1}
$$

This means that TP can be used to express any rewrite $\mathcal{R}$, as long as the type of the candidate syntax admits Term, which is the ability to decompose a datastructure into a universally typed constructor and arguments, and recompose the data-structure back again. Such an approach is not unique to Strafunski. Other typed rewrite systems have also taken the same approach using a universal representation, for example [14]. Using TP, we can give all the type

$$
\text { all }:: \mathrm{TP} \rightarrow \mathrm{TP}
$$

Now it is possible to pass rewrites to all with an arbitrary type, using SYB style generics [8]. Strafunski provides functions for getting into and out of the TP abstraction. Two of the functions for building TP are adhocTP and failTP.

$$
\begin{aligned}
& \text { adhocTP }:: \forall t_{1}\left\langle\text { Term } t_{1}\right\rangle \mathrm{TP} \rightarrow \mathcal{R} t_{1} \rightarrow \mathrm{TP} \\
& \text { failTP }:: \mathrm{TP}
\end{aligned}
$$


Using these combinators, it is possible to use all. We can chain different types of rewrites through our TP abstraction,

\section{all ((failTP 'adhocTP' $r r 1$ ) 'adhocTP' $r r 2$ )}

Here, we have built a TP that can perform two possible rewrites to the immediate children. These two rewrites can be at completely different types. For terms that are not of a matching type, the failTP combinator is applied, causing the rewrite to abort with failure.

The use of generics have significant ramifications for our mission of having a strongly typed DSL for rewrites. There are three shortcomings:

- Even though the generic mechanism itself can be implemented efficiently using a type-safe cast, deep traversals, using recursive invocations of all, become prohibitively expensive, because the universal-type nature of TP results in every single sub-node being considered as a rewrite candidate. For example, when implementing compiler passes, there is a considerable cost to examining every character of every string as a candidate for rewriting.

- The TP type is universal, and rewrites over two completely unrelated syntaxes use the same abstraction. We want the type of our traversal combinators to at least reflect something about the types they operate on. This issue could be at least partially addressed in Strafunski by creatively using a phantom type [15] on TP.

- TP is not a $\mathcal{R}$, therefore not a $\mathcal{T}$ either. We want to build a combinator library around operators on $\mathcal{T}$ (our design decision) and each new user-level type complicates the DSL and the abstractions it is attempting to capture.

\section{Supporting Shallow and Deep Traversals in KURE}

What we want to support in KURE is a version of all that accepts a set of potentially distinctly typed rewrites. Consider a rewrite function, all, to which every relevant correctly typed rewrite is passed explicitly as an element of a tuple.

$$
\text { all :: } \forall t, t_{1}, \ldots, t_{n}\left\langle t_{i} \in \text { childrenOf } t\right\rangle \Rightarrow\left(\mathcal{R} t_{1} \times \mathcal{R} t_{2} \times \ldots \times \mathcal{R} t_{n}\right) \rightarrow \mathcal{R} t
$$

where childrenOf is a function from a type to the set of types of possible children. Implementing all is now a plumbing problem, and straightforward for any specific $t$.

In principle, this idea for implementing all works; the rewriting inside all knows what constructors are being rewritten, so can select the rewrite of the appropriate type. But this version of all has a number of shortcomings. First, the argument to all is difficult to generalize, given that it depends on the number of distinct types contained in the constructors of type $t$. Second, and more importantly, the argument is no longer first class, in the sense that we are building a framework for manipulating rewrites and transforms, and this is a tuple. It would be possible to construct a new sequencing operation over tuples, but this would not make good use of the existing machinery in our DSL. 
A cleaner and preferable approach is to reuse the $\mathcal{R}$ rewrite type as the single argument to all. As discussed in section 4.2 this argument to all can not be parametrically polymorphic, but we can consider how to use our (type) function childrenOf to give a useful and implementable type for all. The question to be considered is can we encode

$$
\left(\mathcal{R} t_{1} \times \mathcal{R} t_{2} \times \ldots \times \mathcal{R} t_{n}\right)
$$

inside

$$
\mathcal{R} t_{G}
$$

What type is $t_{G}$ ? If $t_{G}$ was a common super-type of each of $t_{i}$, then this relationship would hold

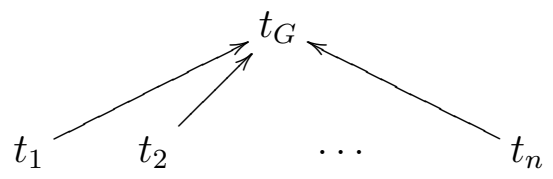

and it would be possible to encode and decode each of the $\mathcal{R} t_{i}$ rewrites inside a single $\mathcal{R} t_{G}$, using this typing relationship. In Haskell, we can model such a typing relationship using sums, so

$$
t_{G}=t_{1}+t_{2}+\ldots+t_{n}
$$

The type of all would therefore be

$$
\text { all :: } \forall t, t_{1}, \ldots, t_{n}\left\langle t_{i} \in \text { childrenOf } t\right\rangle \Rightarrow \mathcal{R}\left(t_{1}+t_{2}+\ldots+t_{n}\right) \rightarrow \mathcal{R} t
$$

We can simplify the type of all, by inventing a type function, $\mathcal{G}$, which maps $t_{i}$ to its super-type $t_{G}$.

$$
\mathcal{G} t_{i}=t_{G}=t_{1}+t_{2}+\ldots+t_{n} \text { where } 0<i \leq n
$$

That is, for each of the type $t_{i}$, we can use $\mathcal{G}$ to look up the generic type, $t_{G}$. Furthermore, if we make $t$ one of the elements in our sum type, then

$$
\mathcal{G} t=\mathcal{G} t_{i}=t_{G}=t+t_{1}+t_{2}+\ldots+t_{n} \text { where } 0<i \leq n
$$

and we can also use the type $t$ to look up the type of $t_{G}$.

This gives us a clean type for all

$$
\text { all }:: \forall t\langle\mathcal{G} t\rangle \Rightarrow \mathcal{R}(\mathcal{G} t) \rightarrow \mathcal{R} t
$$

where $\langle\mathcal{G} t\rangle$ means that $\mathcal{G}$ is defined for $t . \mathcal{R}(\mathcal{G} t)$ replaces TP, as used in Strafunski. Unlike Strafunski, the all used in KURE acts over a specific type, not the universal or generic type. In the same way as in Strafunski uses TP for deep traversals, we use $\mathcal{R}(\mathcal{G} t)$ to provide deep traversal combinators in KURE. 
We will examine the implementation of $\mathcal{G}$ and shallow and deep traversals in section 7 .

$\mathcal{G}$ is the type function that provides our generic mapping to a local universal type, $t_{G}$. By design, we make available to the DSL user the ability to specify what types can map through $\mathcal{G}$ to $t_{G}$. In some cases, there might only be a single type that maps to $t_{G}$, and in larger syntaxes, there might be many. This design flexibility allows the KURE user to craft the type scope of the deep traversals, and for example, avoid considering rewriting strings.

The construction of the sum-type and $\mathcal{R}$ used as an argument to all results in a $\mathcal{T}$ that can represent non type-preserving translations, because

$$
\mathcal{R}\left(t_{1}+t_{2}+\ldots+t_{n}\right)=\mathcal{T}\left(t_{1}+t_{2}+\ldots+t_{n}\right)\left(t_{1}+t_{2}+\ldots+t_{n}\right)
$$

We mitigate against this by providing correct-by-construction promotion functions, and defining translations that are not type-preserving as failed transformations. So a rewrite of type $\mathcal{R} t$ promoted to a $\mathcal{R}(\mathcal{G} t)$ never fails because of incorrect typing, and can be safely combined with other promoted translations, at the $\mathcal{R}(\mathcal{G} t)$ type.

There are other ways of providing deeper traversals, for example, by using fold algebras [16]. Like passing in tuples of $\mathcal{R}$, we could provide combinators that take a representation of a (typed) algebra, and perform a fold-like rewrite. In fact, this could be coded up in KURE. We choose, however, to keep our design simple, where everything is a transform.

Finally, there is one all like combinator we adopt from Strafunski, the crush combinator, which crushes together the results of all the rewrites on the immediate children of a node into one common type. Reusing our type function $\mathcal{G}$, this function would have type.

$$
\text { crush :: } \forall t .\langle\mathcal{G} t \text { and } r \text { is a Monoid }\rangle \Rightarrow \mathcal{T}(\mathcal{G} t) r \rightarrow \mathcal{R} r
$$

\section{Computations Inside KURE}

In KURE, we want to promote small, local rewrites into strategies than can be applied over large syntax terms. We provide a monad, called $\mathcal{M}$, for authoring small rewrites, which also provides some basic services, and a larger abstraction $\mathcal{T}$, which will contain the services of this monad. So, we propose that our rewrites are of the form

$$
\mathcal{T} t_{1} t_{2}=t_{1} \rightarrow \mathcal{M} t_{2}
$$

where $\mathcal{M}$ is our as yet undefined monad. We choose to put all our services into this monad, but there are other choices.

In order to understand the shape of $\mathcal{M}$, we enumerate the capabilities we want to provide in our DSL, towards a generic strategic programming engine in Haskell, and propose how to implement each facility using a specific monad.

- We provide the ability to represent failure, for denoting transformations that fail. This failure does not carry any environment or memory. We implement this using the failure monad. 
- We provide the capability to detect an identity transformation. The Stratego family of rewrite systems offers equality for the cost of a pointer comparison, as provided by the underlying term representation. Knowing that a rewrite performed the identity transformation is critical to a number of important strategy idioms, for example finding a fix-point of a transformation. We implement this status using a count of the non-identity preserving translations performed, and we propagate this using the writer monad.

- By design, we allow the construction of rules that have the ability to generate new global bindings in our candidate syntax. We implement this using the writer monad.

- We provide the ability for a rule to understand and have visibility into its context in a parameterizable manner. We implement this using the reader monad.

- Finally, we provide the ability for KURE users to add other arbitrary capabilities, like unique name generation, and others that may be specific to the underlying grammar.

Combining these capabilities is straightforward using monad transformers [17. After unfolding our monad transformers, we reach a flexible and parameterizable $\mathcal{M}$ monad

$$
\mathcal{M} \alpha=e n v_{\text {read }} \rightarrow m\left(\left(\alpha \times \text { env }_{\text {write }} \times \text { count }_{\text {write }}\right)+\text { Fail }\right)
$$

where $m$ is another monad. In KURE, we implement the $\mathcal{M}$ monad directly using this equation. We will return to the shape of count $_{\text {write }}$ shortly, but we require both env $v_{\text {write }}$ and count write $_{\text {to }}$ be monoids. We explicitly use the reader and writer monad rather than the state monad to allow the possibility of a concurrent implementation of KURE in the future.

In addition to the monadic operators, we provide two additional operations on $\mathcal{T}$ and $\mathcal{M}$, specifically the coercions between the two structures.

$$
\begin{aligned}
\text { translate }::\left(t_{1} \rightarrow \mathcal{M} t_{2}\right) & \rightarrow \mathcal{T} t_{1} t_{2} \\
\text { apply }:: \mathcal{T} t_{1} t_{2} \rightarrow t_{1} & \rightarrow \mathcal{M} t_{2}
\end{aligned}
$$

translate promotes a monadic translation into a $\mathcal{T}$ rewrite, allowing rewrite rules expressed monadically to be constructed. apply allows this monadic code to itself make applications of $\mathcal{T}$ structures; it is the direct equivalent of the ' $<\ldots$. ' syntax in Stratego.

\subsection{Counting Translations and the Equality Flag in $\mathcal{M}$}

An important question to ask after a term has been translated is "has something changed because of this translation?". Comparisons over terms should be cheap if this question is asked often. Checking for equality in Stratego requires a straightforward pointer equality because terms are represented using maximal sharing. In KURE, we want to carefully record an equality approximation, specifically the identity rewrites, and the congruence traversals that transitively only perform identity rewrites. Of course, it is possible for the user to write by hand an 
identity rewrite and generate a false negative, and we intend to perform future measurements to determine how effective our system is in practice. Providing this equality approximation tracking introduces an important challenge.

- The identity rewrite should be tagged as making no change to its argument.

- Also, every translate that contains user-defined code should, by default, be recorded as performing a non-identity translation. That is we assume by default that a function of the form $t_{1} \rightarrow \mathcal{M} t_{2}$ is not simply returning its argument, but has altered the return value in some way.

- However, some translation rules are transparent, in that they perform identity rewrites if the applications of apply inside the translation rule are all transitively the identity rewrite. The conceptual model is that the translation is transparent, and non-identities show through the rule specification.

This is the dilemma. We use translate to promote for both leaf monadic actions, which are not identity translations, and also use translate for building the nodes, which in many cases are identity preserving if the internal calls to apply are also identity preserving. Rather than have two types of translate, we provide a new combinator which can mark a specific translation as transparent.

$$
\text { transparently :: } \forall t_{1}, t_{2} . \mathcal{T} t_{1} t_{2} \rightarrow \mathcal{T} t_{1} t_{2}
$$

By default, translate marks the resulting translation as non-identity preserving by adding one to the count of non-identity sub-translations. transparently is an adjective which modifies translate to not add any additional count value to the non-identity sub-translation count.

Obviously, only an $\mathcal{R}$ can actually be an identity translation, but many combinators are implemented with their most general type (for example <+ and ';'), so we want the ability to also mark these translations as transparent. When transparently is used in a non-type preserving way, the leaf rewrite that actually does the non-type preserving transformation is marked as non-identity, and therefore the whole transformation is also marked as non-identity.

Our use of this checking for equality has an unfortunate consequence; a Translate is not an arrow [18. $\mathcal{T}$ do not hold for the arrow laws, specifically the law

$$
\text { pure id } \gg \text { arr }=\text { arr }
$$

does not hold where arr is a transformation that has been marked as identity preserving. This means that KURE can not use the arrow interface or laws.

\subsection{Implementing Rewrites and Translations}

We have two structures, $\mathcal{T}$, our transformer, and $\mathcal{M}$, our monad, which jointly form the basis of our rewrite system. We used our list of requirements, and the well understood technologies around monads to informally derive a basic implementation for our structures. We now address the question of how we implement $\mathcal{T}$ and $\mathcal{M}$ in Haskell to build a pragmatic DSL for defining rewrites by 
Table 3. Principal Types in KURE

\begin{tabular}{llll}
\hline Name & Type & Implementation & Interface \\
\hline $\mathcal{R}$ e & Rewrite m dec e & Translate m dec e e & Synonym \\
$\mathcal{T}$ e1 e2 & Translate m dec e1 e2 & e1 $\rightarrow$ RewriteM m dec e2 & Abstract \\
$\mathcal{M ~ e ~}$ & RewriteM m dec e & dec $\rightarrow$ m (RewriteStatusM dec e) & Abstract \\
\hline
\end{tabular}

introducing our primary Haskell data-types, and various functions that act as combinators on these functions.

In general, KURE provides syntax rewrites by providing a library of depthless functions, like '; ' and try, then requiring the user to write shallow traversal combinators for their candidate syntax, then providing a library of deep traversal combinator which use the supplied shallow traversal combinators.

Table 3 maps our abstract names onto their type, implementation and interface. Rewrite is a synonym to allow combinators like ';' to be shared between Rewrite and Translate. In Table 3. $\mathrm{m}$ is a monad and dec is the (declaration based) environment. RewriteStatusM can represent success or failure, directly reflecting the internals of the type definition of $\mathcal{M}$.

Table 4 gives the depthless functions provided by KURE, split into functions that perform a monadic computation, functions that generate a Translate, and functions that generate a Rewrite, with brief descriptions. Most of these combinator directly inherite the abilities of their Stratego equivalent. There is also functionality for handling identity detection, provided by changedR, ' . +' and '!->'. changedR turns an identity rewrite into a failed rewrite, mirroring tryR which turns a failing rewrite into an identity rewrite, '. +' which backtracks on identity, and ' !->' which only performs the second rewrite after the first rewrite if the first rewrite was a non-identity rewrite.

\section{Constructing Shallow Traversal Combinators}

In this section, we employ the KURE DSL to build a rewrite DSL over a small syntax, adapted from [19, Grammar 3.1]. We distinguish between DSL code written using KURE and the implementation of KURE itself by placing code fragments that are uses of the KURE DSL (or similar) inside boxes.

We are going to build a set of combinators that act over Stmt and Expr, using Translate and Rewrite. We can use the predefined KURE combinators to write rewrites immediately.

This rewrite attempts to change right associative additions into left associative additions at a single level.

On top of these rewrites, we provide the opportunity to write congruence and shallow traversal combinators, and then take advantage of deep combinators if the DSL user provides the shallow combinators. In KURE we provide two separate styles of structural rewrites, following the conventions of Strafunski. We 
Table 4. Principal Monadic, Translate and Rewrite Functions in KURE

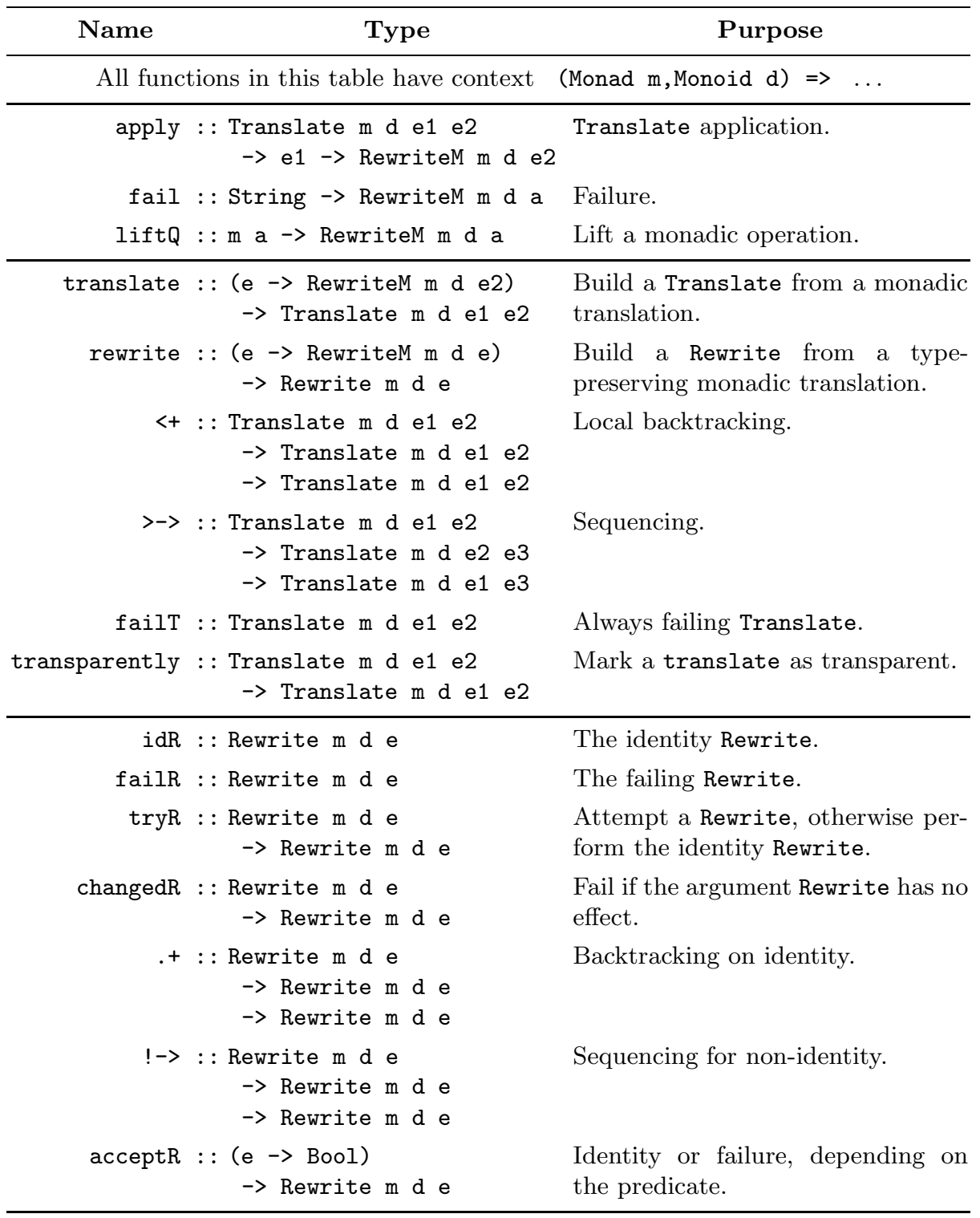

have type-preserving rewrites, suffixed with $\mathrm{R}$, and unifying via a common type rewrites, suffixed with $U$. There are other possible translation patterns possible, for examples based on fold [16]. Our objective is to build a set of translations specific to this syntax, common-up these transformation inside a shallow tree traversal all-like function, and then host our deep generic tree traversals on top of our all combinator. 


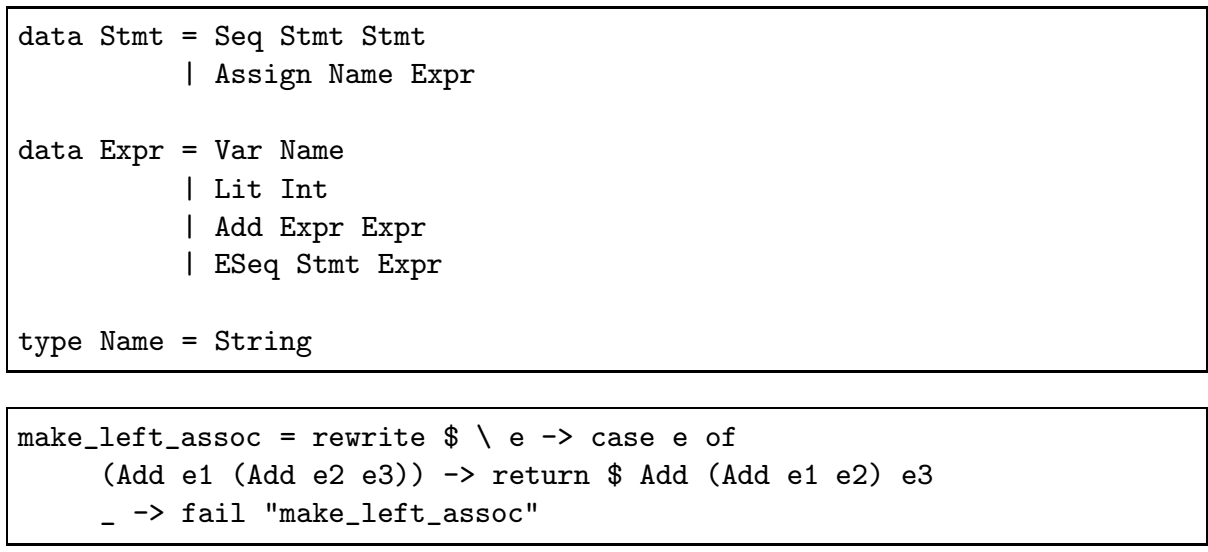

\subsection{Supporting Congruence and Friends}

It is possible to immediately define direct support for congruence and shallow translations in KURE. As an example, we will construct a congruence combinator and the unifying translation for the ESeq constructor. These will have the type.

$$
\begin{aligned}
& \text { eseqR }:: \mathcal{R} \operatorname{Expr} \rightarrow \mathcal{R} \operatorname{Expr} \rightarrow \mathcal{R} \operatorname{Expr} \\
& \text { eseqU }:: \forall u\langle\text { Monoid } u\rangle \Rightarrow \mathcal{T} \operatorname{Expr} u \rightarrow \mathcal{T} \operatorname{Expr} u \rightarrow \mathcal{T} \operatorname{Expr} u
\end{aligned}
$$

Their implementation is tedious but straightforward. The eseqR combinator is marked as transparently rewriting its target, but the esequ is never an identity transformation. Both combinators use apply to invoke the transformation on the arguments of the specific constructor.

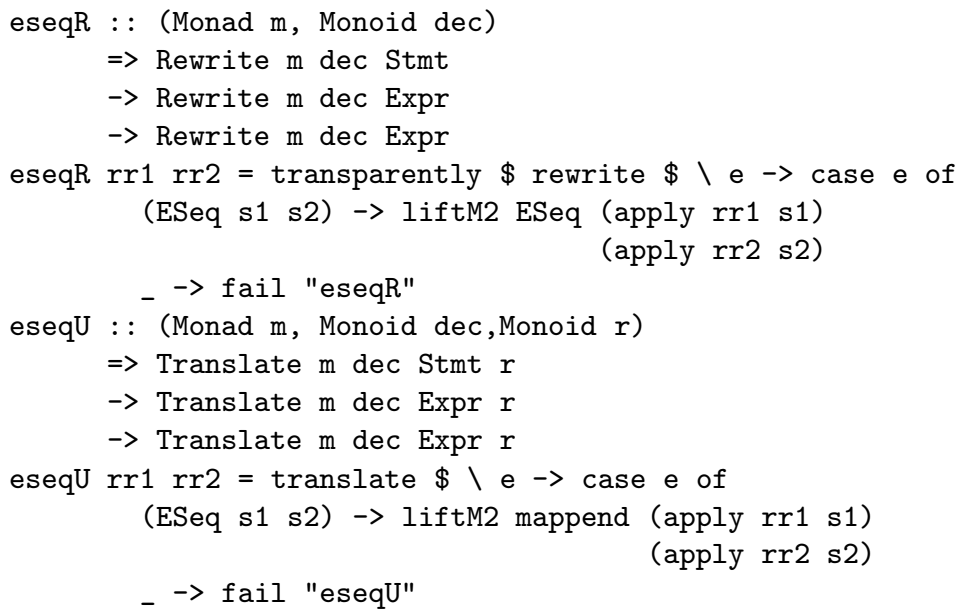


By jumping into the monadic expression world and using apply, writing such congruence combinators is mechanical and prone to cut-and-paste induced errors. The type of the constructors dictates the structure of the implementation of all the functions. The KURE user has the option of being supported in this task with a Template Haskell library, called "KURE your boilerplate" (KYB) which generates these congruence functions automatically from the data structures, performing the tedious task of writing these functions. There may also be good reasons why a library author might want to write these by hand, as we will discuss in section 9, but KYB certainly makes it easier to get KURE working for any specific grammar.

\subsection{Supporting Generic Shallow Term Traversals}

How do we support generic traversals over a syntax tree? In the previous section we implemented congruence, which was supported using multiple Rewrite or Translate arguments, of potentially different types, one per argument of the individual constructor. We want to build generic traversals that take a single Rewrite or Translate. We discussed all in section 5, where we gave it the type

$$
\operatorname{all}:: \forall t\left\langle\mathcal{G} t=t+t_{1}+t_{2}+\ldots\right\rangle \Rightarrow \mathcal{R}(\mathcal{G} t) \rightarrow \mathcal{R} t
$$

The traditional way of capturing the type function $\mathcal{G}$ that is a mapping from $t$ to the sum of possible sub-children type in Haskell is multi-parameter type classes and functional dependencies [20]. However, a more natural implementation route is now possible; we can directly use a type function, as implemented in experimental extension to Haskell called associated type synonyms 3 . We now introduce the relevant part of associated type synonyms, and use it to implement the generic rewrite scheme for KURE.

Type families allow the introduction of data and type declarations inside a class declaration.

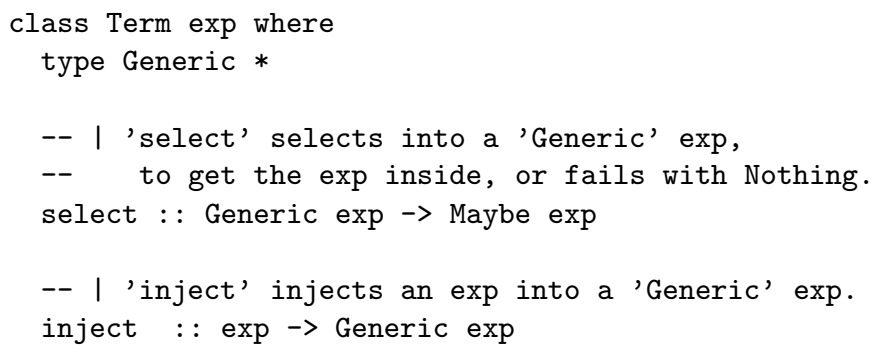

This class declaration creates a type function, Generic which looks like a type synonym inside the class - it does not introduce any constructors or abstraction - but actually provides a configurable function from a type to another type. Unlike traditional type synonyms Generic is only defined for specific instances, specifically instances of our class Term. We have two utility functions. select takes one of our Generic type, and selects a specific type exp, or fails. inject creates something of the Generic type, and can not fail. 
It is now easy to find the Generic type for a supported type, simply using Generic. For our example, we choose to create a new data-type OurGeneric, though we could have chosen to use the Haskell type Either.

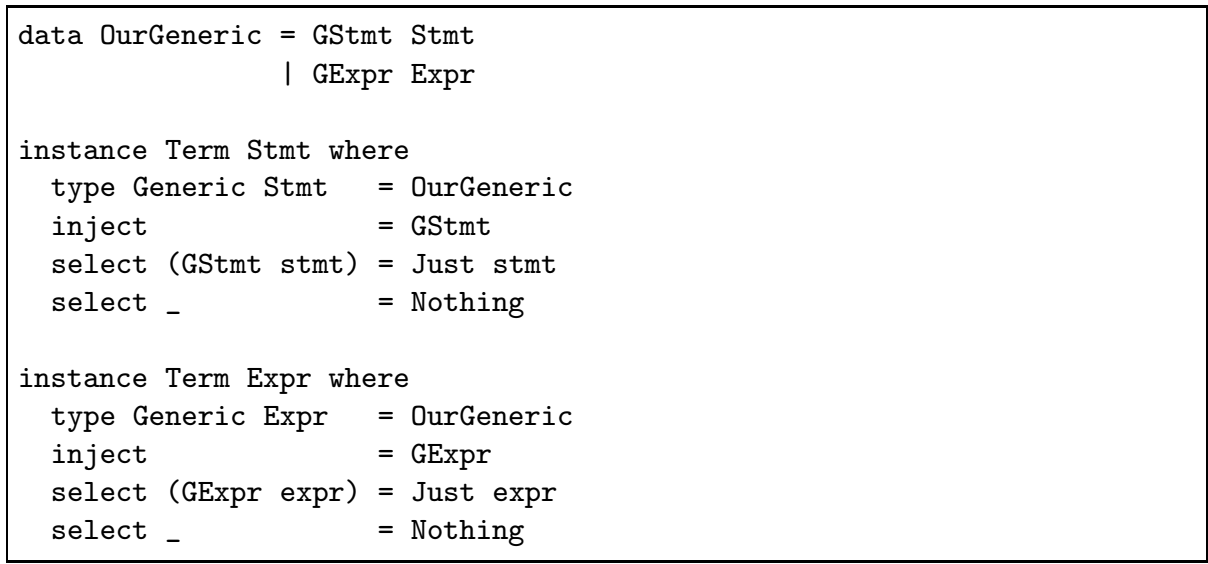

This gives the type equalities

$$
\text { Generic Stmt }=\text { Stmt }+ \text { Expr }=\text { Generic Expr }
$$

Following Strafunski, as well as providing an all combinator in KURE, we also provide a unify function, which requires all (interesting) children to be translatable to a single, unified, monoidal type. Following our naming conventions, we call these two variants allR and crushU. We can now give their interface using class overloading.

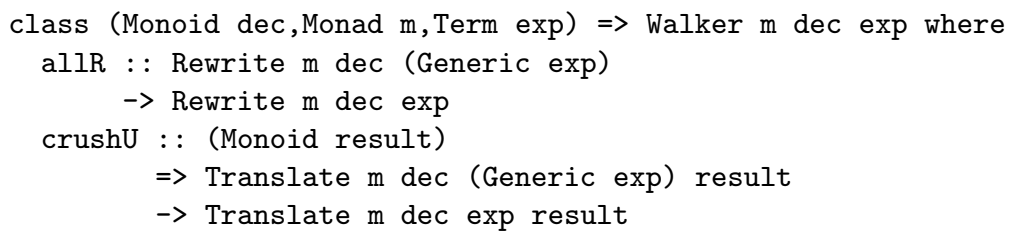

Walker is a multi-parameter type class, which signifies the ability to walk over a specific type. It requires that the type be an instance of type Term, which will provide our generics machinery. allR applies the Generic rewrites to all the chosen children of this node. crushU applied a Generic Translate to a common, monoidal result, to all the interesting children of this node.

Table 5 lists the functions that use the Term typeclass to provide various promotion and extraction functions over the the Translate type, as well as allR and crushU. Term is implicit in the type of allR and crushU, because Walker is a subclass of Term.

If we have provided a complete set of congruence operators (section 7.1 ), then providing the implementation for allR and crushU becomes straightforward. For Expr, our instance can be written as 
Table 5. Shallow Translate Combinators in KURE

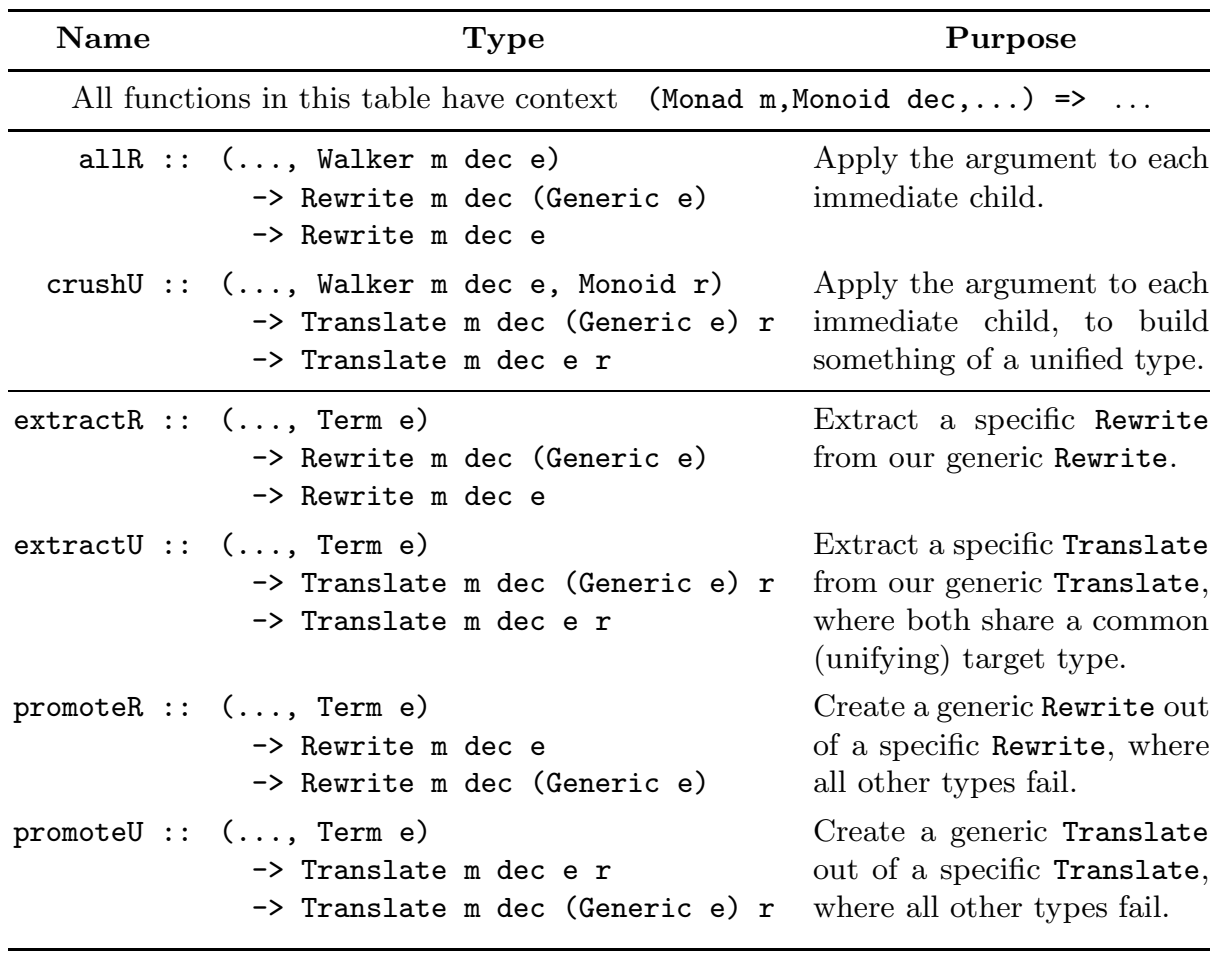

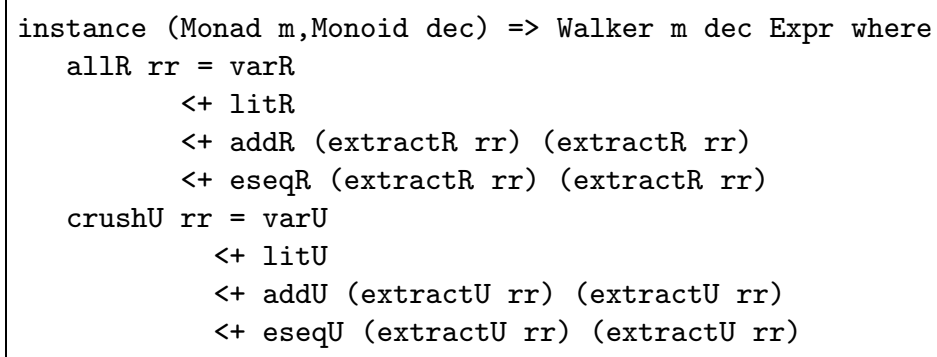

One caveat is we do need to make sure we successfully accept all constructors, including the ones with no interesting arguments, otherwise allR and crushU will unexpectedly fail on these constructors.

\section{Providing Deep Generic Traversal Combinators}

We reach a significant issue when we try to implement deep traversals. Our shallow depth rewrite combinators, like allR act on specific types, but we want to rewrite anything in our Generic type sibling set. If we consider writing a 
topdown rewriter that applies a rewrite at every node in a top-down manner, we appear to want a function of type

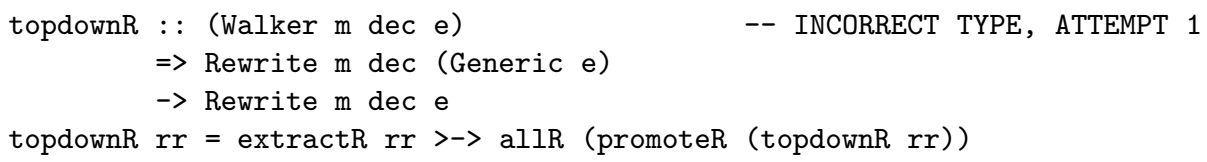

We are confident that Generic e contains all the possible interesting subchildren. However, attempts to compile this function lead to type failures, even though this appears to be a reasonable implementation. We extract the rewrite at the current type, apply it, then rewrite all the children, using topdownR recursively. However, this function is unresolvably ambiguous. The promote allows a single rewrite type to be promoted, and critically, this type is not determinable at compile time for associated type synonyms. This definition actually type-checks for associated data-types, but the use of associated type synonyms is central to our lightweight generic mechanism.

We need to take a different approach. We could take the type from topdown (and all) in Strafunski, which performs the whole traversal at the universal type. In KURE, using our typed 'universal' type, topdown would transliterate into

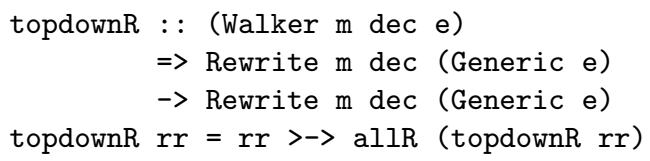

We are now operating at the generic type, hiding any extraction inside rr. However, the type of allR takes a rewrite of type Generic e and returns a rewrite over e. This fails because Generic e does not have the ability to invoke allR directly.

The key to getting topdownR working is empowering Generic e with the same traversal abilities as e, specifically the ability to use Generic to find the generic type. This means that for this function to work, Generic e and e must be of same type. We can achieve this by using the operator provided by associated types, and augmenting the context of deep traversal with the aesthetically pleasing

$$
(. ., \text { Generic e } \sim \text { e) }=>
$$

which means literally that Generic e and e must unify.

Diagrammatically, this means that we now have the following coercion relationship provided by our generic type function, $\mathcal{G}$. 


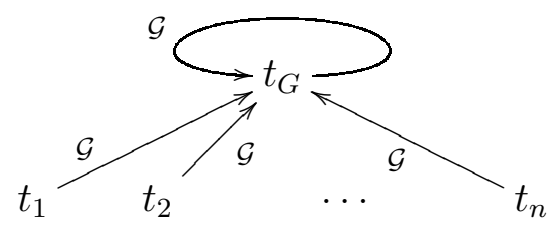

Retrospectively this result is not surprising, in the sense that Generic is being used as a type coercion, and in a custom DSL implementation, the identity coercion would be trivial. Hosting KURE in Haskell has forced the creative use of the type system. Taking this into consideration, we can now give our type and implementation of topdownR, which is

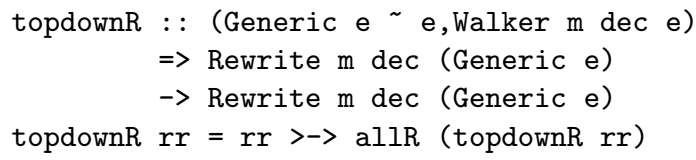

Like in Strafunski, we still return a Rewrite over our universal type, and we can safely use extract when invoking topdownR to build a topdown rewrite strategy at the required type. We can also write the type of topdownR as

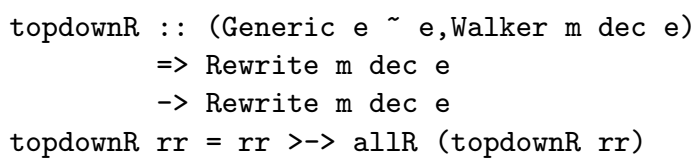

but prefer the former because it expresses explicitly that we are operating over our Generic type.

Table 6] gives a list of the provided deep traversal combinators, all of which use the trick, and have straightforward implementations.

Table 6. Deep Translate Combinators in KURE

\begin{tabular}{ll}
\hline \multicolumn{1}{c}{ Name } & \multicolumn{1}{c}{ Purpose } \\
\hline topdownR & Apply a rewrite in a top down manner. \\
bottomupR & Apply a rewrite in a bottom up manner. \\
alltdR & Apply a rewrite in a top down manner, pruning at successful rewrites. \\
downupR & Apply a rewrite twice, in a top down and bottom up way, using one \\
& single tree traversal. \\
innermostR & A fixed point traversal, starting with the innermost term. \\
& $\quad$ All these functions have type \\
& $\quad \begin{array}{c}\text { (Generic e } \sim \text { e, Walker m dec e) } \\
\quad>\text { Rewrite } \mathrm{m} \mathrm{dec} \mathrm{(Generic} \mathrm{e)}\end{array}$ \\
&
\end{tabular}


In order to provide this reflective Generic ability, we need to provide an instance for Generic e, for each Generic e. To continue our running example, we can have to add an OurGeneric instance for both Term and Walker.

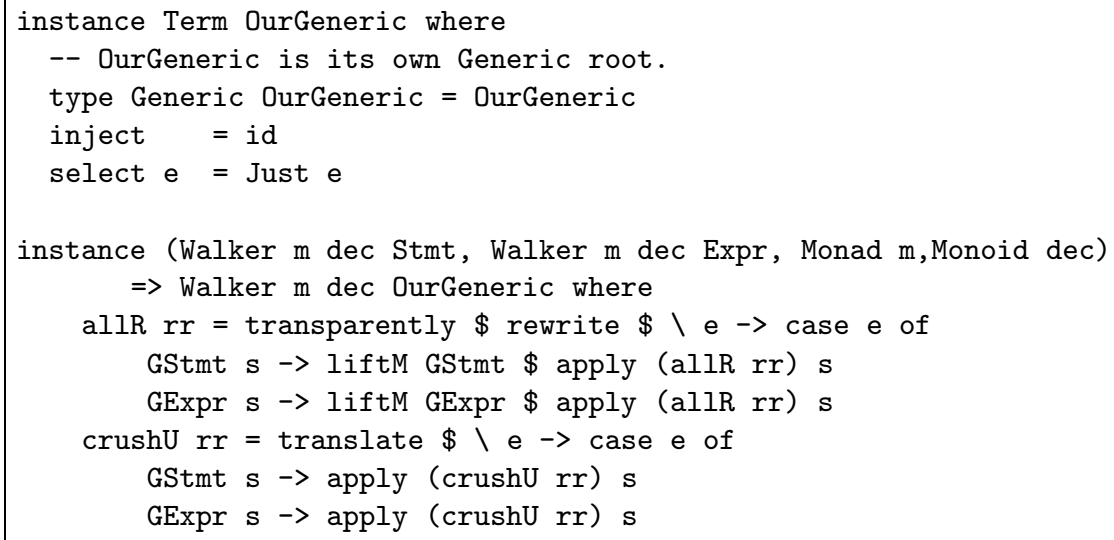

Again, this construction is tedious, but performed only once for each new type of syntax tree. KYB also generates the instance of Walker automatically for the Generic data-type, literally curing more of this boilerplate painfulness. Unfortunately, KYB does not generate the instance for Term because of limitations of the current implementation of Template Haskell.

Though this generic system have proven both useful and sufficient in practice, there are significant technical limitations with our generic mechanism. Specifically, we assume a completely monomorphic syntax tree without any parameterization, and KYB enforces this limitation when generating boilerplate code.

\section{$9 \quad$ Using KURE Extensions}

KURE is intended to help build rewrite engines, based on strategic programming technologies. KURE threads a user-defined local environment though all its transformations and rewrites. This environment must be a monoid. Often, this environment would be (), meaning that the rules are being evaluated in context free manner. Consider an operational semantics where the rules have the form

$$
C \vdash E \rightarrow C \vdash E^{\prime}
$$

$C$ itself does not change, and is read-only. In KURE, we can model $C$ using our environment. For example, $C$ might be the path through the rewrite tree.

$$
C::=\operatorname{root} \mid\langle n\rangle C
$$

We can construct $C$ using the following Haskell code, and define a version of apply that records what edge number we are traversing when applying a Translate. 


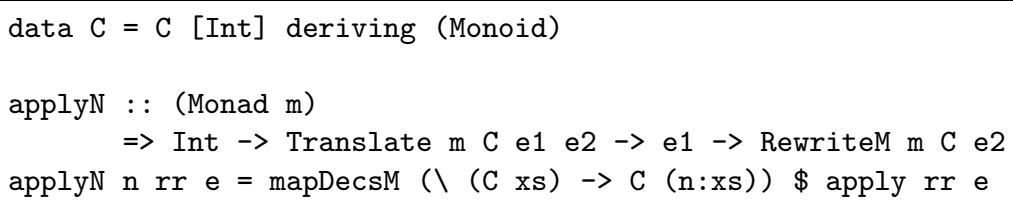

Using applyN, we can rework our congruence methods to use our context, C. For example eseqR would be rewritten

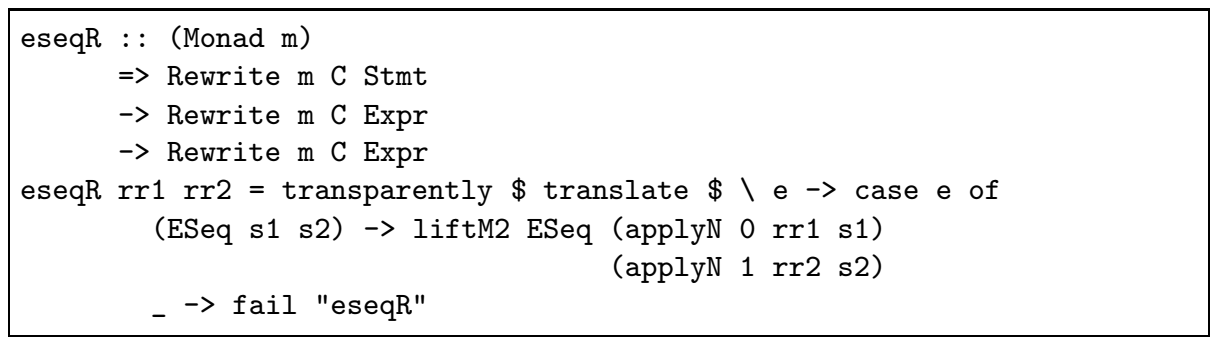

This eseqR performs all the services over Expr that the original eseqR did, but also updates the environment to record the path taken by any tree walker that uses eseqR. With this new location facility provided by the new eseqR (and siblings) we can write a combinator that rewrites at a specific, unique node, using the path through the term tree to find the node.

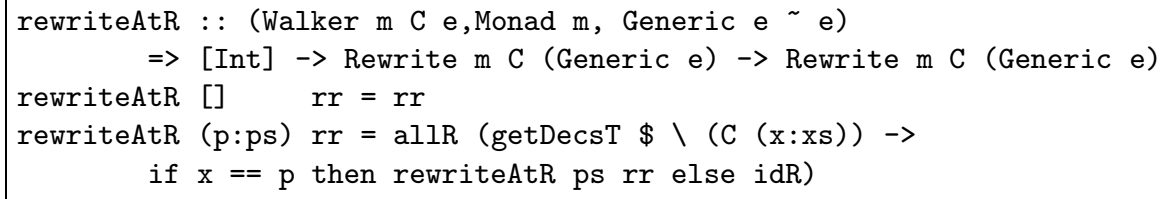

This performs a single rewrite, potentially deep inside the term tree, and gives a small flavor of the flexibility and power of the combinators provided by KURE.

\section{Performance}

In this section we perform some preliminary measurements to evaluate the cost of the flexibility that KURE provides. We know from experience that KURE is reasonably efficient on small and medium sized examples, but for a simple evaluation we implemented Fibonacci using both top-down and bottom-up rewrites in five different systems over a common term representation. In KURE, we implemented Fibonacci using the rewrite fibR.

We also implemented basic arithmetic reductions, as a separate rewrite, and repeatedly use a deep rewrite combinator until no more rewrites can be performed. For bottom-up rewriting, we expressed this using 


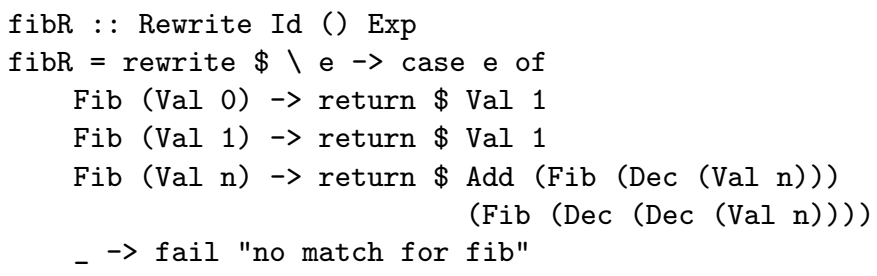

Table 7 gives the preliminary results of our five implementations, measuring wall-clock time in seconds on a $2.5 \mathrm{GHz}$ Intel Mac laptop. Our first two implementations use strategies that can never fail, implemented as simple endomorphic functions. The final three implementations use strategies that can encode failure.

All the implementations use the same basic term rewriting algorithm. The first implementation (Tree Rewrite) uses a straightforward and explicit tree traversal directly coded in Haskell. The second implementation (SYB) used SYB to provide the deep tree traversal. Both of these implementations do not allow for the encoding of failure. The SYB-Maybe and the StrategyLib implementations are both transliterations of the KURE solution. SYB-Maybe encodes failure using the Maybe monad. StrategyLib is a recent version of Strafunski available on hackage.haskell.org. All implementations except KURE compare the result terms after each deep traversal for equality to find a fix-point, while KURE uses the built-in equality rewrite checker, via the '. + ' combinator.

Clearly, KURE is the slowest implementation, and the DSL users pay a cost for the various extra capabilities and counters that KURE provides, up to an order of magnitude of wall clock time over Strafunski. Further investigation will reveal if the costs of KURE can be reduced by using new optimizations inside the Glasgow Haskell compiler. It would be nice if there was a way to completely eliminate the cost of the extra capabilities in KURE which are not being utilized. This brings KURE full circle; it was written to investigate exactly

Table 7. Fibonacci Implemented Using Rewrites

\begin{tabular}{llrrrrrr}
\hline \multirow{2}{*}{ Family } & Test & \multicolumn{3}{c}{ Top Down } & \multicolumn{3}{c}{ Bottom Up } \\
\hline No Failures & Tree Rewrite & 0.018 & 0.157 & 2.024 & 0.010 & 0.075 & 0.987 \\
& SYB & 0.074 & 0.844 & 9.452 & 0.044 & 0.377 & 4.298 \\
\hline Can Fail & KURE & 0.363 & 4.040 & 45.531 & 0.434 & 5.731 & 66.451 \\
& SYB+Maybe & 0.054 & 0.559 & 6.256 & 0.050 & 0.513 & 5.764 \\
& StrategyLib & 0.119 & 1.376 & 15.385 & 0.060 & 0.546 & 6.080 \\
\hline
\end{tabular}


such a transformation 21, inside a more controlled setting than the Glasgow Haskell compiler.

\section{Related Work}

We have already surveyed in some detail two strategic programming systems, Stratego and Strafunski. The widest used and most mature strategy based rewrite system is Stratego 4, which grew out of the work on building a strategy language to translate RML [22], and drew inspiration from ELAN [14], a specification formalism. Stratego is a untyped language for expressing both rewrites and rewrite systems. Strafunski [5] is an implementation of strategic program in Haskell which gives types to strategies, and uses "scrap your boilerplate" generics to provide a universal type, and thus shallow and deep rewrites. Visser [23] is a useful resource as an overview of the discipline.

There have been many, many syntax translators written in Haskell. The Glasgow Haskell compiler itself is an example of a large rewriting optimizer. Another general framework, also being developed at the University of Kansas, is InterpreterLib 24, which uses Modular monadic semantics [25] as its basis for building algebra combinators over co-algebras. There are also systems that express rewrites as extensions to mainstream languages, including Java [26].

\section{Conclusion and Further Work}

We have hosted a strategy based rewrite system in Haskell, using the well understood principles of thinking in terms of types and computations, before implementation. Haskell as a host language worked remarkably well, especially the recent extension for associated type synonyms which were invaluable for finding types for our deeper traversal combinators. KURE as a DSL is clearly aimed at existing Haskell programmers, and requires a significant level of comfort with Haskell before being able to be productive in KURE. This same issue exists with KURE-enabled DSLs that export specific functionality for rewriting a specific syntax. In summary this experiment supports the long held believe that hosted DSLs are great for the users of the host language, and for understanding the intrinsics of a specific DSL, but of questionable general purpose use, except to guide a future stand-alone language.

KURE as a system is in use at the University of Kansas, and forms a base tool for several exploration experiments into rewriting and optimizations. The new Generic mechanism works well in practice, and was a great application of associated type families, but has a number of significant limitations. We will look to lift these restrictions as issues arise in real grammars. We also we want explore the use Haskell's quasi-quoting facility [27. which will allow rewrite rules to be expressed directly in the target syntax as an alternative to working in abstract syntax. 


\section{Acknowledgments}

I would like to thank the members of the Computer Systems Design Laboratory at the Information and Telecommunication Technology Center at the University of Kansas for providing a creative research environment. Specifically, I would like to thank Perry Alexander, Prasad Kulkarni, Vijayanand Manickam, Garrin Kimmell, Nicolas Frisby, as well as Adam Proctor of the University of Missouri. I would also like to thank the referees, for their many detailed and useful suggestions.

\section{References}

1. Peyton Jones, S. (ed.): Haskell 98 Language and Libraries - The Revised Report. Cambridge University Press, Cambridge (2003)

2. Sheard, T., Peyton Jones, S.: Template metaprogramming for Haskell. In: Chakravarty, M.M.T. (ed.) ACM SIGPLAN Haskell Workshop 2002, pp. 1-16. ACM Press, New York (2002)

3. Chakravarty, M.M.T., Keller, G., Jones, S.P.: Associated type synonyms. In: ICFP 2005: Proceedings of the tenth ACM SIGPLAN international conference on Functional programming, pp. 241-253. ACM, New York (2005)

4. Visser, E.: Program transformation with Stratego/XT: Rules, strategies, tools, and systems in StrategoXT-0.9. In: Lengauer, C., Batory, D., Consel, C., Odersky, M. (eds.) Domain-Specific Program Generation. LNCS, vol. 3016, pp. 216-238. Springer, Heidelberg (2004)

5. Lämmel, R., Visser, J.: Typed Combinators for Generic Traversal. In: Krishnamurthi, S., Ramakrishnan, C.R. (eds.) PADL 2002. LNCS, vol. 2257, pp. 137-154. Springer, Heidelberg (2002)

6. Gill, A.: Introducing the Haskell Equational Reasoning Assistant. In: Proceedings of the 2006 ACM SIGPLAN Workshop on Haskell, pp. 108-109. ACM Press, New York (2006)

7. Hutton, G., Meijer, E.: Monadic parsing in Haskell. Journal of Functional Programing 8(4), 437-444 (1998)

8. Lämmel, R., Peyton Jones, S.: Scrap your boilerplate: a practical design pattern for generic programming. ACM SIGPLAN Notices 38(3), 26-37 (2003); Proc. of the ACM SIGPLAN Workshop on Types in Language Design and Implementation (TLDI 2003)

9. Bravenboer, M., van Dam, A., Olmos, K., Visser, E.: Program transformation with scoped dynamic rewrite rules. Fundamenta Informaticae 69(1-2), 123-178 (2006)

10. Dolstra, E.: Functional stratego. In: Visser, E. (ed.) Proceedings of the Second Stratego Users Day (SUD 2001), pp. 10-17 (2001)

11. Dolstra, E.: First-class rules and generic traversal for program transformation languages. Master's thesis, Utrecht University, Utrecht, The Netherlands, INF/SCR2001-15 (August 2001)

12. Dolstra, E., Visser, E.: First-class rules and generic traversal. Technical Report UUCS-2001-38, Institute of Information and Computing Sciences, Utrecht University, Utrecht, The Netherlands (2001)

13. Hall, C., Hammond, K., Jones, S.P., Wadler, P.: Type classes in Haskell. ACM Transactions on Programming Languages and Systems 18, 241-256 (1996) 
14. Borovansky, P., Kirchner, C., Kirchner, H., Ringeissen, C.: Rewriting with strategies in ELAN: A functional semantics. International Journal of Foundations of Computer Science 1, 69-95 (2001)

15. Leijen, D., Meijer, E.: Domain specific embedded compilers. In: 2nd USENIX Conference on Domain Specific Languages (DSL 1999), Austin, Texas, October 1999, pp. 109-122 (1999)

16. Lämmel, R., Visser, J.: Type-safe functional strategies. In: Scottish Functional Programming Workshop (2000)

17. Liang, S., Hudak, P., Jones, M.: Monad transformers and modular interpreters. In: ACM (ed.) Conference record of POPL 1995, 22nd ACM SIGPLAN-SIGACT Symposium on Principles of Programming Languages, San Francisco, California, pp. 333-343. ACM Press, New York (1995)

18. Hughes, J.: Generalising monads to arrows. Science of Computer Programming 37, 67-111 (2000)

19. Appel, A.W.: Modern Compiler Implementation in Java, 2nd edn. Cambridge University Press, Cambridge (2002)

20. Jones, M.P., Diatchki, I.S.: Language and program design for functional dependencies. In: Haskell 2008: Proceedings of the first ACM SIGPLAN symposium on Haskell, pp. 87-98. ACM, New York (2008)

21. Gill, A., Hutton, G.: The worker/wrapper transformation. Journal of Functional Programming 19(2), 227-251 (2009)

22. Visser, E., Benaissa, Z., Tolmach, A.: Building program optimizers with rewriting strategies. In: Proceedings of the third ACM SIGPLAN International Conference on Functional Programming (ICFP 1998), pp. 13-26. ACM Press, New York (1998)

23. Visser, E.: A survey of rewriting strategies in program transformation systems. In: Gramlich, B., Lucas, S. (eds.) Workshop on Reduction Strategies in Rewriting and Programming (WRS 2001), Utrecht, The Netherlands. Electronic Notes in Theoretical Computer Science, vol. 57. Elsevier Science Publishers, Amsterdam (2001)

24. Weaver, P., Kimmell, G., Frisby, N., Alexander, P.: Constructing language processors with algebra combinators. In: GPCE 2007: Proceedings of the 6th international conference on Generative programming and component engineering, pp. 155-164. ACM, New York (2007)

25. Harrison, W.L., Kamin, S.N.: Metacomputation-based compiler architecture. In: Mathematics of Program Construction, pp. 213-229 (2000)

26. Balland, E., Moreau, P.E., Reilles, A.: Rewriting strategies in Java. Electron. Notes Theor. Comput. Sci. 219, 97-111 (2008)

27. Mainland, G.: Why it's nice to be quoted: quasiquoting for Haskell. In: Haskell 2007: Proceedings of the ACM SIGPLAN workshop on Haskell, pp. 73-82. ACM, New York (2007) 